\title{
Apatinib sensitizes chemoresistant NSCLC cells to doxetaxel via regulating autophagy and enhances the therapeutic efficacy in advanced and refractory/recurrent NSCLC
}

\author{
RONG HU ${ }^{1,2^{*}}$, TAO LI $^{1 *}$, KAIYUAN HUI $^{3 *}$, ZI CHEN $^{1}$, NAN WANG $^{1}$, \\ XINGPING WU ${ }^{2}$, LINYANG GE ${ }^{1}$ and LINFU ZHOU ${ }^{1,4}$
}

\begin{abstract}
${ }^{1}$ Department of Respiratory and Critical Care Medicine, The First Affiliated Hospital of Nanjing Medical University, Nanjing, Jiangsu 210029; ${ }^{2}$ Department of Respiratory and Critical Care Medicine; ${ }^{3}$ Tumor Laboratory, Department of Oncology, The First People's Hospital of Lianyungang, Lianyungang, Jiangsu 222006; ${ }^{4}$ Laboratory of Respiratory Diseases, Institute of Integrative Medicine, Nanjing Medical University, Nanjing, Jiangsu 210029, P.R. China
\end{abstract}

Received December 11, 2019; Accepted July 21, 2020

DOI: $10.3892 / \mathrm{mmr} .2020 .11492$

\begin{abstract}
The prognosis of advanced non-small cell lung cancer (NSCLC) is poor; therefore, identifying novel treatment strategies for patients with NSCLC is important. The present study aimed to investigate the efficacy of apatinib plus docetaxel vs. docetaxel alone, as well as their effects on regulating autophagy markers in patients with advanced NSCLC. Furthermore, it was evaluated whether apatinib sensitized chemoresistant NSCLC cells to docetaxel via regulating autophagy. A total of 39 patients with advanced NSCLC were consecutively enrolled and treated with apatinib plus docetaxel $(n=19)$ or docetaxel alone $(n=20)$ for four treatment cycles. The treatment response, adverse events and expression levels of autophagy markers [(light chain $3 \alpha$ (LC3A) and Beclin-1] were evaluated in tumor samples, which were obtained via biopsy, before treatment and after 2-cycle treatment. In addition, in a mechanistic in vitro experiment, apatinib, docetaxel, the autophagy activator rapamycin and the autophagy inhibitor 3-methyladenine (3-MA) were used to treat docetaxel-resistant A549 (A549/DTX) cells alone or in various combinations.
\end{abstract}

Correspondence to: Professor Linfu Zhou, Department of Respiratory and Critical Care Medicine, The First Affiliated Hospital of Nanjing Medical University, 300 Guangzhou Road, Nanjing, Jiangsu 210029, P.R. China

E-mail:1fzhou@njmu.edu.cn

${ }^{*}$ Contributed equally

Abbreviations: NSCLC, non-small cell lung cancer; VEGFR-2, vascular endothelial growth factor receptor-2; RECIST, Response Evaluation Criteria in Solid Tumors; CR, complete remission; PR, partial remission; $\mathrm{SD}$, stable disease; $\mathrm{PD}$, progressive disease; ORR, overall remission rate; DCR, disease control rate; AE, adverse event

Key words: apatinib, docetaxel, advanced NSCLC, autophagy, chemoresistance
The expression levels of LC3A, Beclin-1, poly (ADP) ribose polymerase (PARP) and phosphorylated (p)-AKT were detected via western blotting, while the cell apoptosis rate was detected with an Annexin V/PI assay. The overall remission rate (37 vs. $10 \% ; \mathrm{P}=0.047)$ and disease control rate $(84$ vs. $45 \%$; $\mathrm{P}=0.011$ ) were increased in the Apatinib plus docetaxel group compared with the Docetaxel group. Most of the adverse events were mild and tolerable, and there was no difference between the two groups except for total hypertension and hand-foot syndrome, which were higher in the Apatinib plus docetaxel group). Compared with the levels prior to treatment, Beclin-1 and LC3A remained unchanged post-treatment in the Apatinib plus docetaxel group, while they were increased in the Docetaxel group. Docetaxel increased LC3A, Beclin-1 and $\mathrm{p}$-AKT expression levels, PARP cleavage and the cell apoptosis rate in A549/DTX cells, and rapamycin further enhanced, while 3-MA reduced these effects of docetaxel. Moreover, apatinib repressed LC3A, Beclin-1, p-AKT expression levels and promoted the cell apoptosis rate in A549/DTX cells and docetaxel-treated A549/DTX cells. In conclusion, apatinib synergize the effect of docetaxel in treating patients with advanced NSCLC and chemoresistant NSCLC cells via inhibiting autophagy.

\section{Introduction}

Lung cancer is the leading cause of cancer-associated mortality worldwide, among which non-small cell lung cancer (NSCLC) accounts for $>85 \%$ (1). Although numerous improvements have been achieved in therapeutic methods including surgical strategy, neo-adjuvant therapy, targeted drugs and molecular markers, the prognosis of NSCLC remains far from satisfaction, particularly for advanced cases who fail to benefit from tumor resection (2). Furthermore, chemotherapy-based treatment remains the primary option for patients with advanced NSCLC, while a proportion of patients experience chemoresistance, post-response progression and metastasis after chemotherapy, which represents a considerable bottleneck for their clinical treatment (3). Therefore, it is an urgent challenge 
to identify novel and effective treatment options to improve the prognosis of these patients with advanced NSCLC.

Apatinib, as a novel small-molecule anti-angiogenesis agent, exerts a favorable anti-tumor effect in terms of inhibiting the proliferation and migration of endothelial cells, reducing angiogenesis and micro-vascularization of tumors via selectively binding or inhibiting vascular endothelial growth factor receptor-2 (VEGFR-2) and suppressing its downstream signal transduction pathways (4,5). Despite increased evidence of the efficacy of apatinib in treating advanced cancer types, including gastric cancer, colorectal cancer and ovarian cancer, its application in NSCLC has rarely been investigated and only a few studies with small sample sizes have been performed (6-9). Apatinib has been reported to attenuate multi-chemotherapy drug resistance in several cancer types using in vitro experiments and may enhance the efficacy of chemotherapy in treating patients with advanced cancer types in clinical settings $(10,11)$. Moreover, apatinib is able to regulate autophagy in serval cancer types, including anaplastic thyroid cancer, colon cancer and osteosarcoma, and autophagy is considered a key factor in NSCLC chemoresistance and metastasis (12). Based on the abovementioned findings, it was hypothesized that apatinib may be able to synergize the efficacy of chemotherapeutics in the treatment of NSCLC via regulating autophagy.

Therefore, the present study aimed to investigate the efficacy and safety of apatinib plus docetaxel vs. docetaxel alone, and their effects on regulating autophagy markers in patients with advanced NSCLC. In addition, it was evaluated whether apatinib was able to sensitize the cells to docetaxel-induced apoptosis in chemoresistant NSCLC cells via regulating autophagy.

\section{Materials and methods}

Patients. A total of consecutive 39 patients (age, 47-75 years; 26 male patients and 13 female patients) with advanced NSCLC, who underwent apatinib plus docetaxel treatment or docetaxel alone treatment, were retrospectively enrolled in The First Affiliated Hospital of Nanjing Medical University between January 2017 and December 2018. The inclusion criteria were as follows: i) Diagnosed with primary NSCLC at stage IV or recurrent metastatic NSCLC refractory/intolerant to standard therapy; ii) age $\geq 18$ years; iii) a baseline Eastern Cooperative Oncology Group performance status score of 0-1 (13); iv) adequate hematologic, hepatic and renal functions; v) cessation of other anti-tumor therapies for $\geq 1$ month prior to enrollment; vi) presence of $\geq 1$ measurable lesion defined by Response Evaluation Criteria in Solid Tumors version 1.1 (RECIST 1.1) (14); and vii) a survival prognosis of $>3$ months. Furthermore, the exclusion criteria were as follows: i) Previously treated with immunotherapy; ii) untreated metastases of the central nervous system; iii) uncontrolled blood pressure with medication (>140/90 $\mathrm{mmHg}$ ); iv) serious infection or autoimmune disease; v) bleeding tendency; and vi) hepatopathy, nephropathy, cardiopathy, respiratory disease or uncontrollable diabetes.

The present study was approved by the Ethics Committee of The First Affiliated Hospital of Nanjing Medical University, and all patients provided written informed consent prior to enrollment.

Treatment and assessment. Patients were divided into two groups: The Apatinib plus docetaxel group $(n=19)$ and the Docetaxel group $(n=20)$. In the Apatinib plus docetaxel group, patients received apatinib (500 mg/day, orally; Heng Rui Pharmaceutics; www.hrs.com.cn) and docetaxel $\left(60 \mathrm{mg} / \mathrm{m}^{2}\right.$, intravenously; Heng Rui Pharmaceutics) on day 1 every 3 weeks for a total of four treatment cycles. In the Docetaxel group, patients received docetaxel $\left(60 \mathrm{mg} / \mathrm{m}^{2}\right.$, intravenously; Heng Rui Pharmaceutics) on day 1 every 3 weeks for a total of four cycles. The treatment response was evaluated after four cycles of treatment, referring to the RECIST 1.1 criteria, as follows: Complete remission (CR), partial remission (PR), stable disease (SD) or progressive disease. Then, the overall remission rate (ORR) was calculated as $\mathrm{CR}+\mathrm{PR}$, and the disease control rate (DCR) was calculated as $\mathrm{CR}+\mathrm{PR}+\mathrm{SD}$. In addition, adverse events (AE) were recorded and evaluated according to the National Cancer Institute Common Terminology Criteria for AE (version 4.0; www.meddramsso.com).

Immunohistochemistry (IHC) analysis. It has been reported that apatinib regulates autophagy in various cancer types and autophagy is considered as a key factor in chemotherapy drug resistance $(12,15-17)$. Therefore, the expression levels of autophagy markers, light chain $3 \alpha$ (LC3A) and Beclin-1, were detected in tumor tissues pre-treatment and post-treatment using IHC analysis. Lung tumor tissues, obtained via biopsy pre-treatment (before treatment) and post-treatment [after two cycles of treatment ( 42 days)], were available from only three patients in the Apatinib plus docetaxel group and three patients in the Docetaxel group, and these tissues were subjected to IHC analysis.

The tumor tissue section (thickness, $4 \mu \mathrm{m}$ ), which was fixed with $4 \%$ paraformaldehyde (Sigma-Aldrich; Merck KGaA) at $4^{\circ} \mathrm{C}$ for $24 \mathrm{~h}$ and embedded in paraffin (Sigma-Aldrich; Merck $\mathrm{KGaA}$ ), was deparaffinized, rehydrated and subjected to antigen retrieval, which was followed by blocking with $10 \%$ goat serum (Sigma-Aldrich; Merck $\mathrm{KGaA}$ ) at $37^{\circ} \mathrm{C}$ for $1 \mathrm{~h}$ and $0.3 \% \mathrm{H}_{2} \mathrm{O}_{2}$ to block non-specific binding and peroxidase activity, respectively. Subsequently, primary anti-Beclin-1 (1:100; cat. no. bsm-33315M) and anti-LC3A antibodies (1:100; cat. no. bsm-33309M; both Beijing Biosynthesis Biotechnology Co., Ltd.) were added and incubated at $4^{\circ} \mathrm{C}$ overnight. Subsequently, the samples were incubated with secondary horseradish peroxidase (HRP)-conjugated goat anti-rabbit IgG (H+L) antibody (1:10,000; cat. no. A27036; Invitrogen; Thermo Fisher Scientific, Inc.) at $37^{\circ} \mathrm{C}$ for $60 \mathrm{~min}$. The tissue sections were stained with diaminobenzidine (Sigma-Aldrich; Merck $\mathrm{KGaA}$ ) at $37^{\circ} \mathrm{C}$ for $5 \mathrm{~min}$ and counterstained with $0.5 \%$ hematoxylin (Sigma-Aldrich; Merck KGaA) at room temperature for $5 \mathrm{~min}$. The IHC staining results were observed under a Nikon ECLIPSE E200 light microscope (Nikon Corporation; magnification, x400), and assessed for staining intensity and density of positively stained cells according to a previously described method (18), with an IHC staining score of 0-12 for each section.

Cell culture. Human NSCLC cells (wild-type A549) and docetaxel-resistant A549 cells (A549/DTX) were purchased 
from the Type Culture Collection of the Chinese Academy of Sciences. Cells were cultured in 90\% RPMI-1640 medium (Gibco; Thermo Fisher Scientific, Inc.) supplemented with 10\% FBS (Zhejiang Tianhang Biological Technology Co., Ltd.) and $100 \mathrm{U} / \mathrm{ml}$ penicillin (Sigma-Aldrich; Merck KGaA) and streptomycin (Sigma-Aldrich; Merck $\mathrm{KGaA}$ ) at $37^{\circ} \mathrm{C}$ in a humidified atmosphere containing $5 \% \mathrm{CO}_{2}$.

Determination of A549/DTX cells. To determine the docetaxel resistance of A549/DTX cells, 0, 2.5, 5, 10 or $20 \mu \mathrm{M}$ docetaxel (Abmole Bioscience Inc.) was added to treat A549/DTX cells and wild-type A549 cells for $24 \mathrm{~h}$ at $37^{\circ} \mathrm{C}$. Cell viability was detected using Cell Counting Kit-8 (Sigma-Aldrich; Merck KGaA) according to the manufacturer's instructions at a wavelength of $450 \mathrm{~nm}$. Then, the relative cell viability was calculated by referring to the viability of cells treated with $0 \mu \mathrm{M}$ docetaxel in each cell type.

Docetaxel, rapamycin and 3-methyladenine (3-MA) treatments of A549/DTX cells and subsequent analyses. In order to investigate whether autophagy promotes docetaxel resistance in A549/DTX cells, $10 \mu \mathrm{M}$ docetaxel (Abmole Bioscience Inc.), $20 \mu \mathrm{M}$ autophagy activator rapamycin (Sigma-Aldrich; Merck KGaA) and $50 \mathrm{mM}$ autophagy inhibitor 3-MA (Sigma-Aldrich; Merck KGaA) were used alone or in combination to treat the cells simultaneously at $37^{\circ} \mathrm{C}$. The concentration of docetaxel was selected based on the $\mathrm{IC}_{50}$ value of docetaxel in A549/DTX cells reported in a previous study (19). The concentrations of rapamycin and 3-MA were in reference to our previous study (20).

After $48 \mathrm{~h}$ of treatment, the protein expression levels of LC3A, Beclin-1, poly(ADP) ribose polymerase (PARP) and phosphorylated (p)-AKT were determined via western blot analysis in each group of cells. Moreover, the apoptotic rate in each group of cells was determined with an Annexin V/PIdouble staining kit (Calbiochem; Merck KGaA) using a FACSCanto II flow cytometer (BD Biosciences), according to the manufacturer's protocol. The data was analyzed using FlowJo software (version 7.6; BD Biosciences) (21).

Apatinib and docetaxel treatment of A549/DTX cells and subsequent experiments. In order to investigate whether apatinib synergizes the anti-cancer effect of docetaxel on A549/DTX cells via regulating autophagy, $10 \mu \mathrm{M}$ apatinib (Selleck Chemicals) and $10 \mu \mathrm{M}$ docetaxel (Abmole Bioscience, Inc.) were used alone or in combination to treat the cells at $37^{\circ} \mathrm{C}$. After $48 \mathrm{~h}$ of incubation, the protein expression levels LC3A, Beclin-1, PARP and p-AKT were determined via western blot analysis, while the apoptosis rate was determined using a Annexin V/PI double staining kit (Calbiochem; Merck KGaA), according to the manufacturer's protocol, in each group of cells.

Apatinib and docetaxel treatments of wild-type A549 cells and subsequent assays. In order to investigate whether apatinib synergizes the anti-cancer effect of docetaxel in wild-type A549 cells via regulating autophagy, $10 \mu \mathrm{M}$ apatinib (Selleck Chemicals) and $10 \mu \mathrm{M}$ docetaxel (Abmole Bioscience, Inc.) were used to treat wild-type A549 cells alone or in combination at $37^{\circ} \mathrm{C}$. After $48 \mathrm{~h}$ of treatment, the expression levels of LC3A and Beclin-1 were determined via western blot analysis, and the cell apoptosis rate was determined with an Annexin V/PI double staining kit (Calbiochem; Merck KGaA), according to the manufacturer's protocol, in each group of cells.

Western blot analysis. After isolation of total protein with RIPA lysis and extraction buffer (Thermo Fisher Scientific, Inc.), the protein concentration was quantified with the Pierce $^{\mathrm{TM}}$ BCA Protein assay kit (Thermo Fisher Scientific, Inc.). Subsequently, $20 \mu \mathrm{g}$ protein samples were separated using 4-20\% SDS-PAGE (Nanjing KeyGen Biotech Co., Ltd.) and then transferred onto Immobilon ${ }^{\circledR}-\mathrm{P}$ Transfer membranes (Merck KGaA). After blocking with 5\% non-fat milk for $2 \mathrm{~h}$ at $37^{\circ} \mathrm{C}$, membranes were incubated with primary antibodies, including anti-Beclin-1 (1:1,000; cat. no. bs-1353R; Beijing Biosynthesis Biotechnology Co., Ltd.), anti-LC3A (1:1,000; cat. no. bsm-33309M; Beijing Biosynthesis Biotechnology Co., Ltd.), anti-p-AKT antibody (1:1,000; cat. no. 4058; Cell Signaling Technology, Inc.), anti-PARP antibody (1:1,000; cat. no. 9532; Cell Signaling Technology, Inc.) and anti- $\beta$-actin antibody (1:10,000; cat. no. sc-58679; Santa Cruz Biotechnology, Inc.), overnight at $4^{\circ} \mathrm{C}$. Subsequently, the membranes were incubated with rabbit anti-mouse IgG-HRP (1:5,000; cat. no. sc-358917; Santa Cruz Biotechnology, Inc.) or goat anti-rabbit IgG-HRP (1:5,000; cat. no. sc-2004; Santa Cruz Biotechnology, Inc.) for $1 \mathrm{~h}$ at room temperature. The bands were visualized using ECL kit (Nanjing KeyGen Biotech Co., Ltd.) followed by exposure to X-ray film (Kodak).

Reverse transcription-quantitative $(R T-q) P C R$. After the extraction of total RNA using PureZOL RNA isolation reagent (Bio-Rad Laboratories, Inc.), it was reverse transcribed to cDNA using the ReverTra Ace qPCR RT kit (Toyobo Life Science) at $37^{\circ} \mathrm{C}$ for $15 \mathrm{~min}$. Subsequently, qPCR was performed using the KOD SYBR qPCR mix (Toyobo Life Science) to quantify the mRNA expression levels of LC3A and Beclin-1. The thermocycling conditions used for qPCR were as follows: Initial denaturation at $98^{\circ} \mathrm{C}$ for $2 \mathrm{~min}$; 40 cycles of denaturation at $98^{\circ} \mathrm{C}$ for $10 \mathrm{sec}$ and annealing and extension at $61^{\circ} \mathrm{C}$ for $30 \mathrm{sec}$. The quantified results were determined using the $2^{-\Delta \Delta \mathrm{Cq}}$ method with $\beta$-actin as the internal reference (22).

The sequences of the primers were as follows: LC3A forward, 5'AGCGAGTTGGTCAAGATCATC3' and reverse, 5'GGTTTCCTGGGAGGCGTAGA3'; Beclin-1 forward, 5'TCAGAGATACCGACTTGTTCCTTAC3' and reverse, 5'ACTGCCTCCTGTGTCTTCAATC3'; and $\beta$-actin forward, 5'TCGTGCGTGACATTAAGGAGAA3' and reverse, 5'AGG AAGGAAGGCTGGAAGAGT3'.

Statistical analysis. The experiments were performed in triplicate. Statistical analyses were performed using SPSS software version 22.0 (IBM Corp.), and plots were generated using GraphPad Prism Software version 7.00 (GraphPad Software, Inc.). Data are presented as the mean \pm standard deviation for continuous variables and $\mathrm{n}(\%)$ for categorical variables. Comparisons between two independent groups of continuous data were analyzed using an unpaired Student's t-test. Comparisons between two independent groups of categorical data were analyzed usingthe $\chi^{2}$ test or Fisher's exact test. Comparison between two paired groups was performed 
using the paired t-test. Multiple comparisons among groups was performed using one-way ANOVA followed by Tukey's multiple-comparisons test. $\mathrm{P}<0.05$ was considered to indicate a statistically significant difference.

\section{Results}

Basic characteristics of patients. A total of 14 males and five females, aged 47-75 years, including six patients with adenocarcinomas and 13 with squamous cell carcinomas, were included in the Apatinib plus docetaxel group. Moreover, 12 males and eight females, aged 48-75 years, including 10 patients with adenocarcinomas and 10 with squamous cell carcinomas, were included in Docetaxel group. There was no difference in sex, age or histological type between the two groups (all $\mathrm{P}>0.05)$.

Treatment efficacy. After the four cycles of treatment, the CR was the same ( 0 vs. $0 \% ; \mathrm{P}=1.000)$, while the ORR (37 vs. $10 \%$; $\mathrm{P}=0.047$ ) and DCR (84 vs. $45 \% ; \mathrm{P}=0.011$ ) were increased in the Apatinib plus docetaxel group compared with the Docetaxel group, respectively (Table I). These results indicated that apatinib plus docetaxel was more efficient compared with docetaxel alone in treating patients with advanced NSCLC.

AEs occurrence. The occurrences of total hypertension (58 vs. $0 \%, \mathrm{P}<0.001$ ) and total hand-foot syndrome (26 vs. $0 \%$, $\mathrm{P}=0.014)$ were significantly higher in the Apatinib plus docetaxel group compared with those in the Docetaxel group, respectively. However, no differences in any other AEs, such as neutropenia, were present between the Apatinib plus docetaxel group and the Docetaxel group (Table II). Moreover, all the AEs in the two groups were mild and tolerable.

Autophagy markers. Representative IHC staining images are presented in Fig. 1A. Semi-quantitative IHC scoring demonstrated that in the Docetaxel group, Beclin-1 $(\mathrm{P}<0.01)$ and LC3A $(\mathrm{P}<0.05)$ expression levels were increased after two cycles of treatment compared with the levels prior to treatment (Fig. 1B and C), while in the Apatinib plus docetaxel group, the expression levels remained similar after two cycles of treatments compared with the pre-treatment score (both $\mathrm{P}>0.05$; Fig. 1B and C). These results suggested that apatinib attenuated autophagy in advanced NSCLC induced by docetaxel for $\geq 2$ cycles (treatment duration, 42 days).

Validation of docetaxel resistance of A549/DTX cells. A549/DTX cells demonstrated increased relative cell viability compared with wild-type A549 cells under 2.5, 5, 10 and $20 \mu \mathrm{M}$ docetaxel treatment $(\mathrm{P}<0.05)$, which suggested that A549/DTX cells were docetaxel resistant (Fig. S1).

Combination treatments on A549/DTX cells. A549/DTX cells were treated with $10 \mu \mathrm{M}$ docetaxel, $20 \mu \mathrm{M}$ rapamycin and $50 \mathrm{mM}$ 3-MA alone or in combination (Fig. 2). Docetaxel treatment increased the protein expression levels of LC3A, Beclin-1, p-AKT and PARP in A549/DTX cells compared with the control $(\mathrm{P}<0.05$; Fig. $2 \mathrm{~A}$ and $\mathrm{D})$. Rapamycin plus docetaxel treatments further increased the protein expression levels of LC3A, Beclin-1, p-AKT and PARP compared with
Table I. Treatment response between the Apatinib plus docetaxel group and the Docetaxel group.

\begin{tabular}{lccc}
\hline Parameter & $\begin{array}{c}\text { Docetaxel } \\
\text { group }\end{array}$ & $\begin{array}{c}\text { Apatinib plus } \\
\text { docetaxel group }\end{array}$ & P-value \\
\hline CR, n (\%) & $0(0)$ & $0(0)$ & 1.000 \\
PR, n (\%) & $2(10)$ & $7(37)$ & 0.047 \\
SD, n (\%) & $7(35)$ & $9(47)$ & 0.433 \\
PD, n (\%) & $11(65)$ & $3(16)$ & 0.011 \\
ORR, n (\%) & $2(10)$ & $7(37)$ & 0.047 \\
DCR, n (\%) & $9(45)$ & $16(84)$ & 0.011 \\
\hline
\end{tabular}

Docetaxel group, $n=20$. Apatinib plus docetaxel group, $n=19$. CR, complete remission; PR, partial remission; $\mathrm{SD}$, stable disease; PD, progressive disease; ORR, overall remission rate; DCR, disease control rate.

docetaxel treatment in A549/DTX cells ( $\mathrm{P}<0.05$; Fig. 2A), while 3-MA plus docetaxel treatments reduced LC3A, Beclin-1, p-AKT and PARP expression levels compared with docetaxel treatment in A549/DTX cells in A549/DTX cells $(\mathrm{P}<0.05$; Fig. 2D). Furthermore, the mRNA expression levels of LC3A and Beclin-1 exhibited similar trends to the protein expression levels in most groups of A549/DTX cells $(\mathrm{P}<0.05$; Fig. 2B, C, E and F).

The cell apoptotic rate was decreased in the Rapamycin group $(\mathrm{P}<0.01)$, while it was increased in 3-MA group $(\mathrm{P}<0.01)$ and Docetaxel group $(\mathrm{P}<0.001)$ compared with the Control group (Fig. 3A and B). In addition, the cell apoptotic rate was reduced in the Docetaxel plus Rapamycin group $(\mathrm{P}<0.05)$, but it was enhanced in the Docetaxel plus 3-MA group compared with Docetaxel group $(\mathrm{P}<0.05$; Fig. $3 \mathrm{~A}$ and $\mathrm{B})$. These results suggested that autophagy attenuated the effect of docetaxel to induce apoptosis in A549/DTX cells.

Apatinib synergized the effect of docetaxel in the treatment of A549/DTX cells via regulating autophagy. Apatinib $(10 \mu \mathrm{M})$ and docetaxel $(10 \mu \mathrm{M})$ were used to treat A549/DTX cells alone or in combination. Apatinib reduced the protein expression levels of LC3A, Beclin-1 and p-AKT in docetaxel-treated A549/DTX cells $(\mathrm{P}<0.05)$, while the expression of PARP was not notably affected (P>0.05; Fig. 4A). Moreover, the mRNA expression levels of LC3A and Beclin-1 exhibited similar trends to their protein expression in each group of A549/DTX cells $(\mathrm{P}<0.05$; Fig. 4B and $\mathrm{C})$.

The cell apoptotic rate was increased in Docetaxel alone and Apatinib alone groups compared with the control group (both $\mathrm{P}<0.001$ ), but also in the Apatinib plus docetaxel group compared with the Docetaxel group $(\mathrm{P}<0.05)$ and Apatinib group $(\mathrm{P}<0.01$; Fig. $4 \mathrm{D}$ and $\mathrm{E})$. Thus, it was indicated that apatinib synergized the effect of docetaxel in treating A549/DTX cells via suppressing autophagy.

Synergism of apatinib with docetaxel in treating wild-type A549 cells. Apatinib $(10 \mu \mathrm{M})$ and docetaxel $(10 \mu \mathrm{M})$ were used to treat wild-type A549 cells alone or in combination. In wild-type A549 cells, docetaxel had a less pronounced effect on enhancing the autophagy markers LC3A and 
Table II. Adverse events between the Apatinib plus docetaxel group and the Docetaxel group.

\begin{tabular}{|c|c|c|c|c|c|c|c|}
\hline \multirow[b]{2}{*}{ Parameter } & \multicolumn{3}{|c|}{ Docetaxel group } & \multicolumn{3}{|c|}{ Apatinib plus docetaxel group } & \multirow[b]{2}{*}{$P$-value ${ }^{a}$} \\
\hline & Grade $1-2$ & Grade 3-4 & Total & Grade $1-2$ & Grade 3-4 & Total & \\
\hline Neutropenia, n (\%) & $15(75)$ & $0(0)$ & $15(75)$ & $16(84)$ & $0(0)$ & $16(84)$ & 0.476 \\
\hline Thrombocytopenia, n (\%) & $4(20)$ & $0(0)$ & $4(20)$ & $5(26)$ & $0(0)$ & $5(26)$ & 0.640 \\
\hline Anemia, n (\%) & $4(20)$ & $0(0)$ & $4(20)$ & $2(11)$ & $0(0)$ & $2(11)$ & 0.412 \\
\hline Hypertension, n (\%) & $0(0)$ & $0(0)$ & $0(0)$ & $10(53)$ & $1(5)$ & $11(58)$ & $<0.001$ \\
\hline Hand-foot syndrome, n (\%) & $0(0)$ & $0(0)$ & $0(0)$ & $5(26)$ & $0(0)$ & $5(26)$ & 0.014 \\
\hline Fatigue, n (\%) & $4(20)$ & $0(0)$ & $4(20)$ & $7(37)$ & $0(0)$ & $7(37)$ & 0.243 \\
\hline Proteinuria, n (\%) & $0(0)$ & $0(0)$ & $0(0)$ & $3(16)$ & $0(0)$ & $3(16)$ & 0.064 \\
\hline Hemorrhage, n (\%) & $0(0)$ & $0(0)$ & $0(0)$ & $1(5)$ & $0(0)$ & $1(5)$ & 0.297 \\
\hline Oral mucositis, n (\%) & $1(5)$ & $0(0)$ & $1(5)$ & $0(0)$ & $1(5)$ & $1(5)$ & 1.000 \\
\hline Nausea, n (\%) & $16(80)$ & $0(0)$ & $16(80)$ & $15(79)$ & $0(0)$ & $15(79)$ & 0.935 \\
\hline Diarrhea, n (\%) & $0(0)$ & $0(0)$ & $0(0)$ & $0(0)$ & $0(0)$ & $0(0)$ & 1.000 \\
\hline Hyperbilirubinemia, n (\%) & $4(20)$ & $0(0)$ & $4(20)$ & $3(16)$ & $0(0)$ & $3(16)$ & 0.732 \\
\hline Elevated transaminase, $\mathrm{n}(\%)$ & $2(10)$ & $0(0)$ & $2(10)$ & $2(11)$ & $0(0)$ & $2(11)$ & 0.957 \\
\hline
\end{tabular}

${ }^{\mathrm{a} C}$ Comparison of total adverse events. Docetaxel group, $\mathrm{n}=20$. Apatinib plus docetaxel group, $\mathrm{n}=19$.

A

A
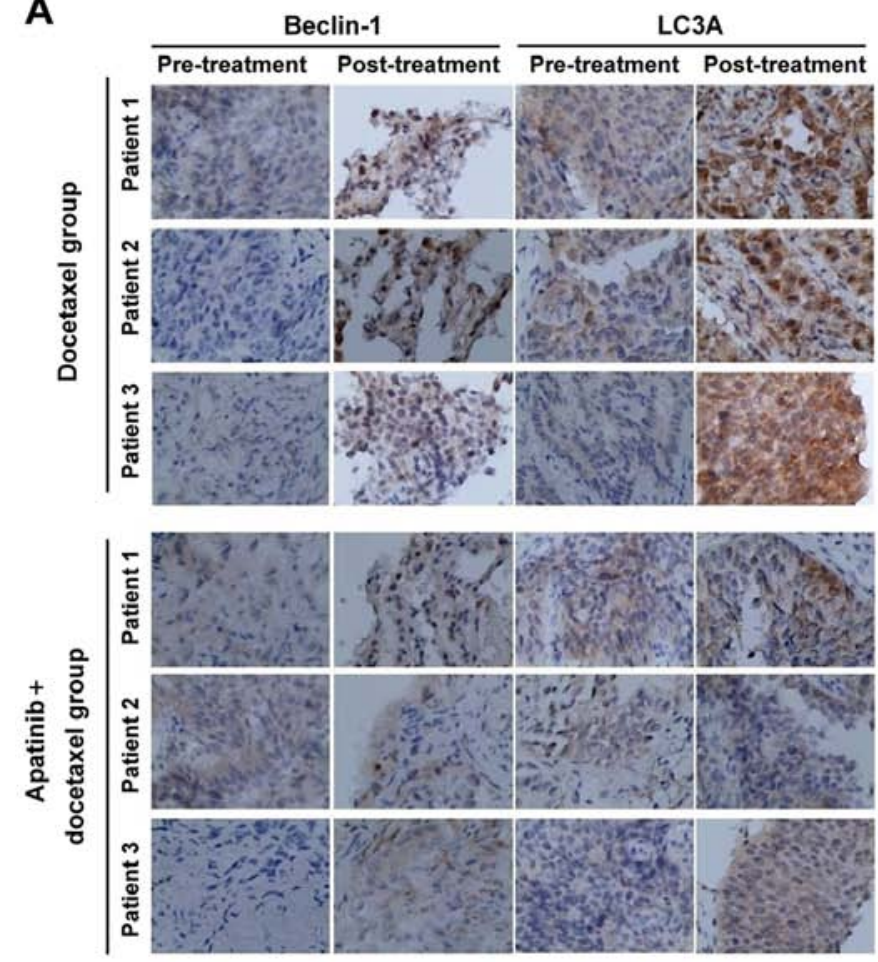

B

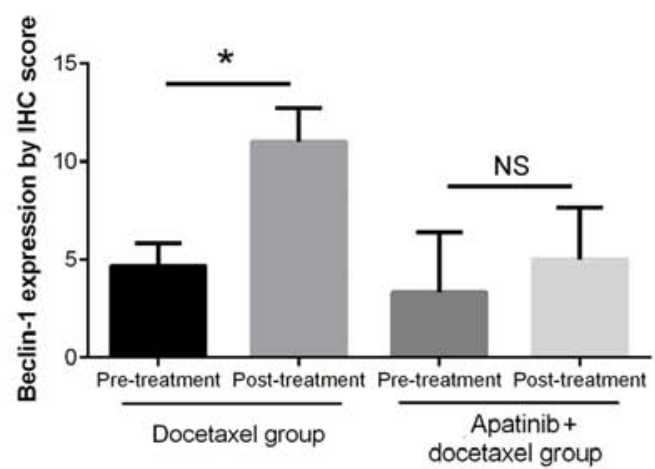

C

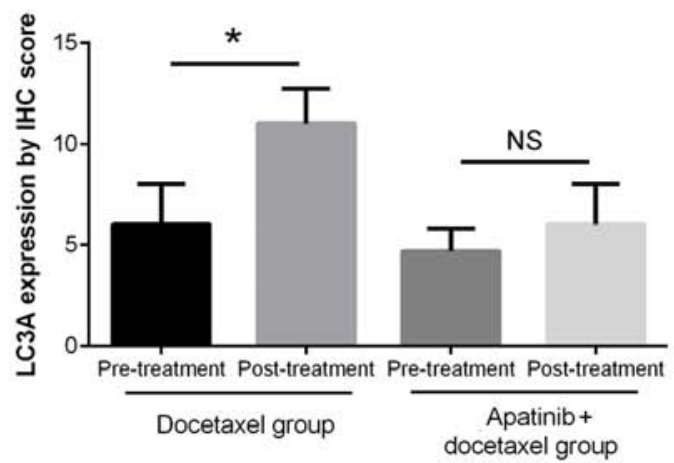

Figure 1. Beclin-1 and LC3A expression levels pre- and post-treatment. (A) IHC staining images for Beclin-1 and LC3A pre- and post-treatment in the Apatinib plus docetaxel group and Docetaxel group (magnification, x400). (B) IHC semi-quantitative score of Beclin-1 expression. (C) IHC semi-quantitative score of LC3A expression. " P<0.05. IHC, Immunohistochemistry; LC3A, light chain $3 \alpha$; NS, not significant.

Beclin-1, while apatinib exhibited a notable effect to repress autophagy markers (Fig. 5A). Furthermore, the cell apoptosis rate was significantly increased in the Docetaxel alone and Apatinib alone groups compared with the control group (both $\mathrm{P}<0.001$ ), and also in the Apatinib plus docetaxel group compared with the Docetaxel group $(\mathrm{P}<0.05)$ and Apatinib group $(\mathrm{P}<0.05$; Fig. $5 \mathrm{~B}$ and $\mathrm{C})$. These results suggested that apatinib exhibited a synergistic effect with docetaxel in the treatment of wild-type A549 cells.

\section{Discussion}

The present study demonstrated that: i) Apatinib plus docetaxel improved the treatment efficacy and attenuated autophagy 


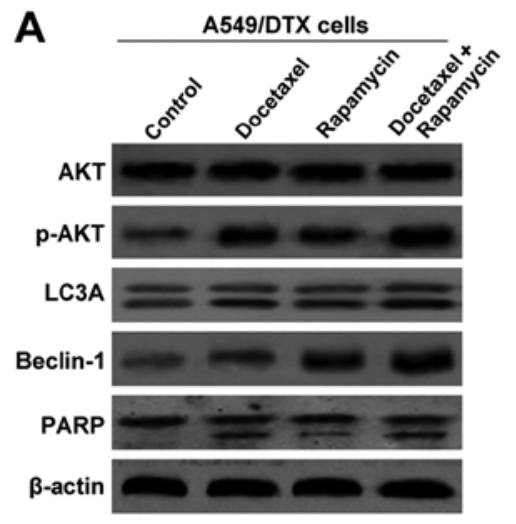

B C
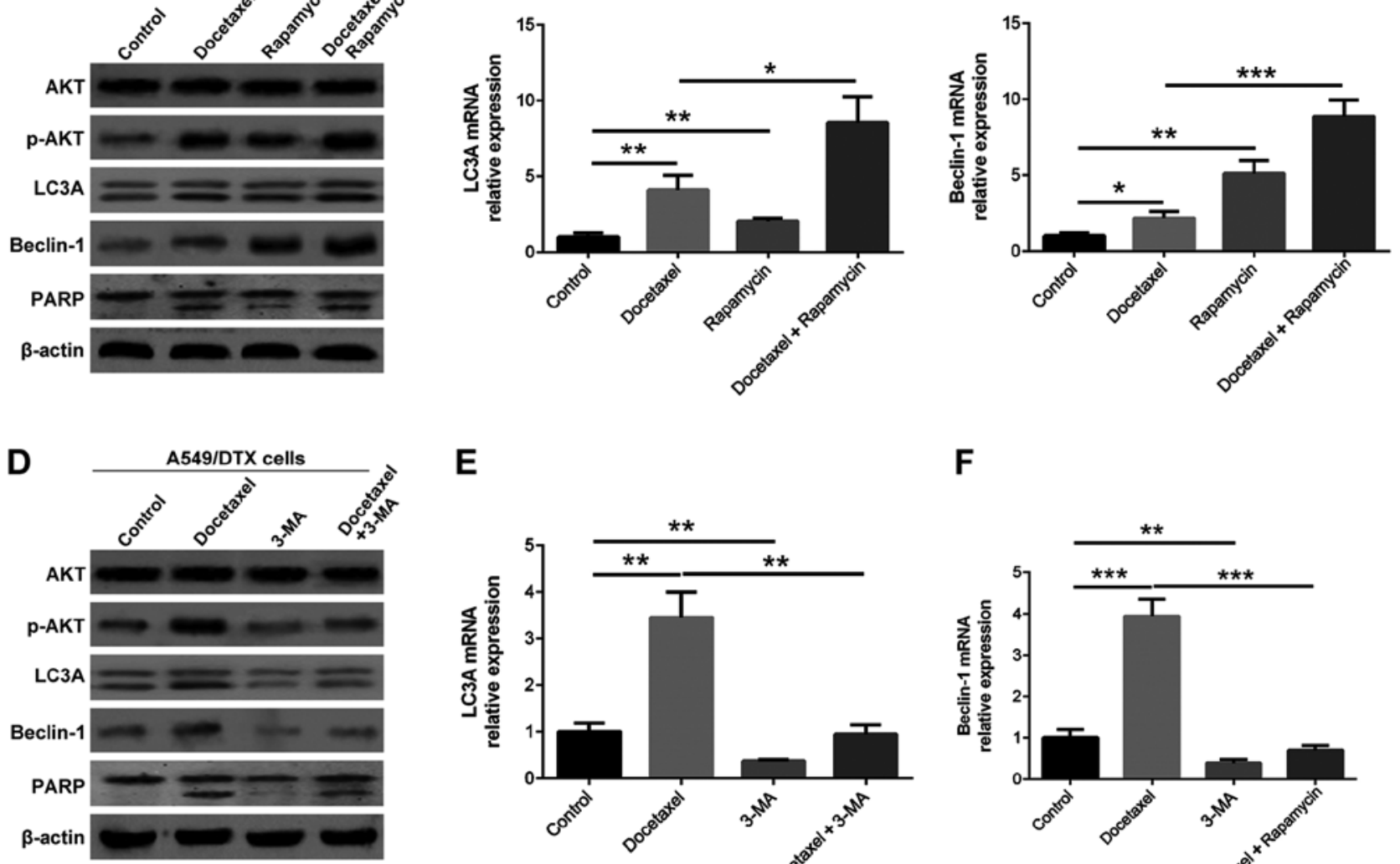

E

$\mathbf{F}$
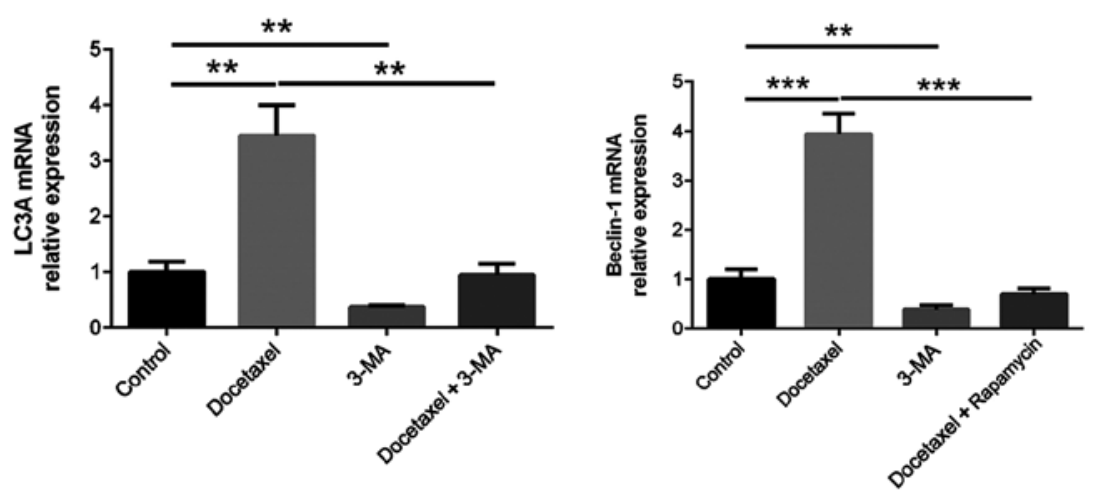

Figure 2. LC3A, Beclin-1, AKT, p-AKT and PARP expression levels following treatment with rapamycin, 3-MA and docetaxel. (A) LC3A, Beclin-1, AKT, p-AKT and PARP protein expression levels after rapamycin and docetaxel treatment. (B) LC3A and (C) Beclin-1 mRNA expression levels after rapamycin and docetaxel treatment. (D) LC3A, Beclin-1, AKT, p-AKT and PARP protein expression levels after 3-MA and docetaxel treatment. (E) LC3A and (F) Beclin-1 mRNA expression levels after 3-MA and docetaxel treatment. ${ }^{*} \mathrm{P}<0.05,{ }^{* *} \mathrm{P}<0.01$ and ${ }^{* * * *} \mathrm{P}<0.001$. LC3A, light chain $3 \alpha$; p-AKT, phosphorylated AKT; 3-MA, 3-methyladenine; PARP, poly (ADP) ribose polymerase.

A
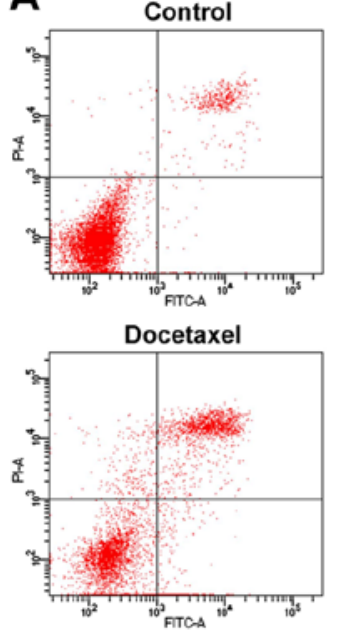

Rapamycin

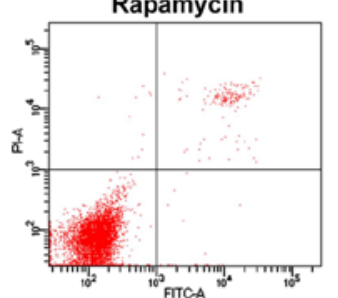

Docetaxel plus Rapamycin

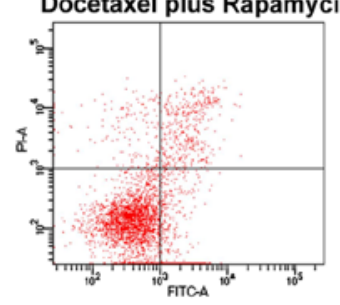

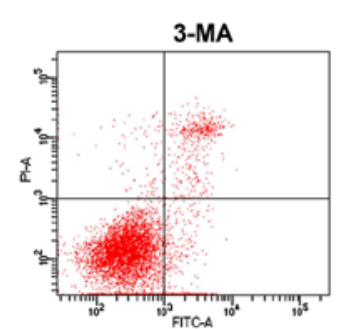

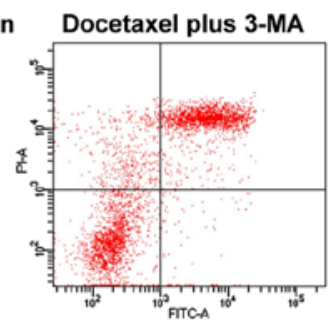

B

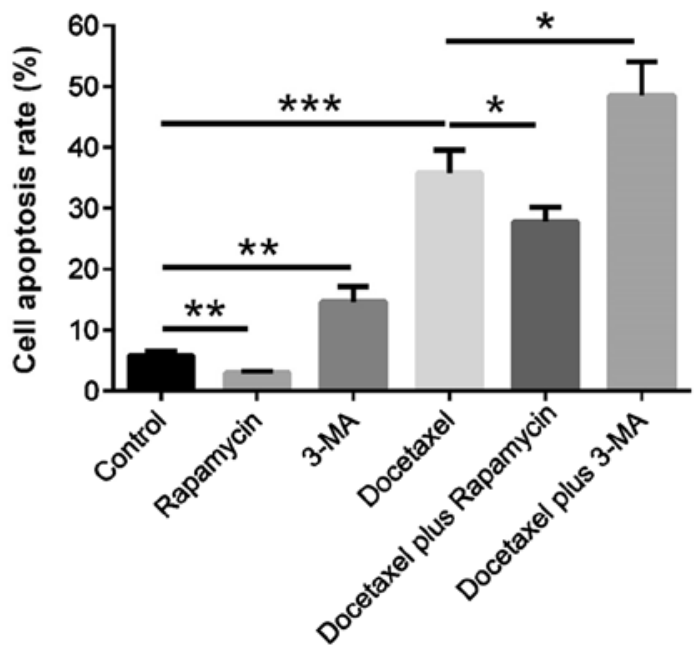

Figure 3. Cell apoptotic rate of A549/DTX cells affected by rapamycin, 3-MA and docetaxel treatment. (A) Representative flow cytometry images of cell apoptosis and (B) bar graph presenting the cell apoptosis rate following treatment with rapamycin, 3-MA and docetaxel. ${ }^{*} \mathrm{P}<0.05,{ }^{* *} \mathrm{P}<0.01$ and ${ }^{* * *} \mathrm{P}<0.001$. 3-MA, 3-methyladenine.

compared with docetaxel alone in patients with advanced NSCLC; ii) autophagy reduced the cytotoxic effect of docetaxel on A549/DTX cells; and iii) apatinib sensitized A549/DTX cells to the cytotoxic effect of docetaxel by repressing autophagy. 
A

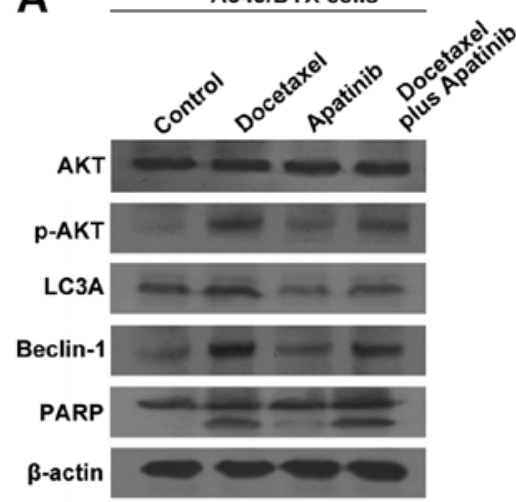

B

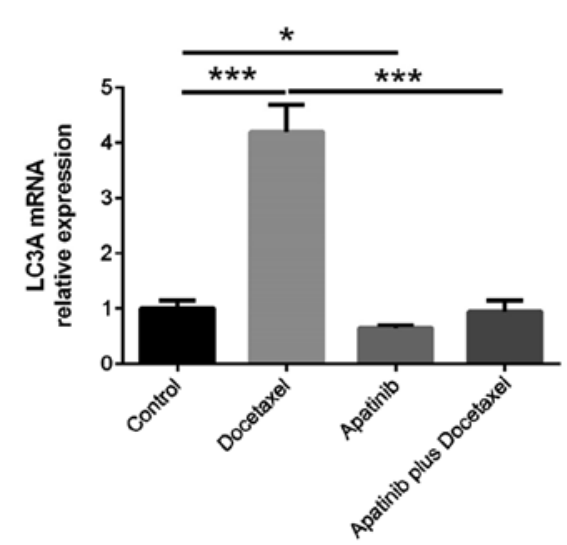

C

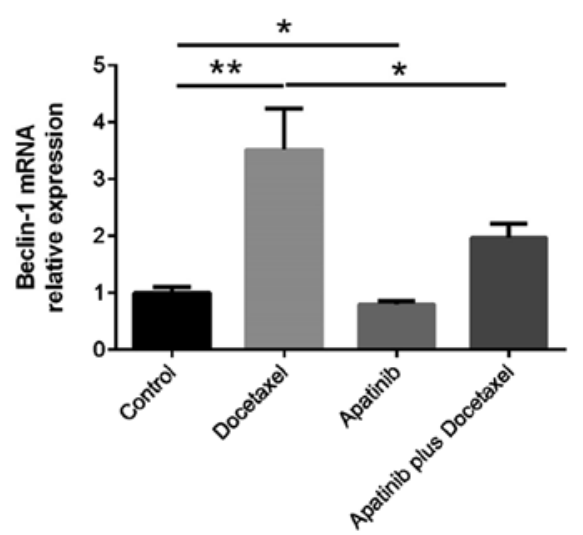

D
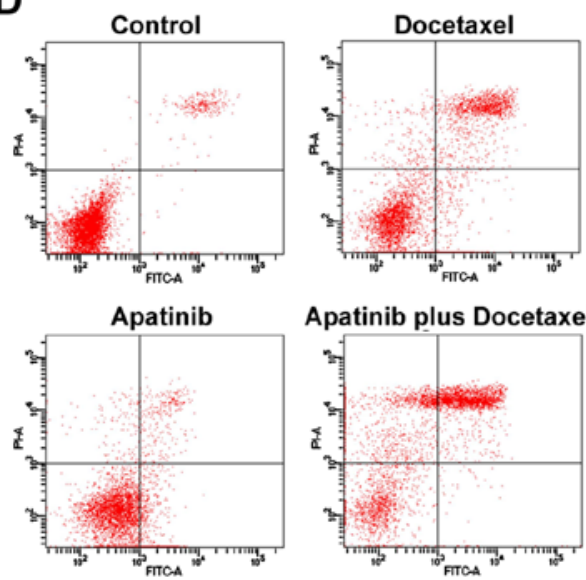

Apatinib plus Docetaxel

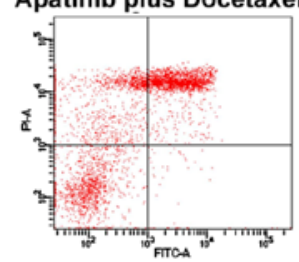

E

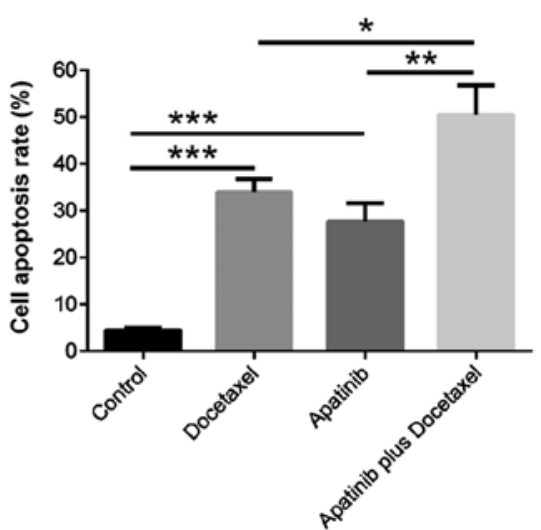

Figure 4. LC3A, Beclin-1, AKT, p-AKT and PARP expression levels and cell apoptosis rate of A549/DTX cells following apatinib and docetaxel treatment. (A) LC3A, Beclin-1, AKT, p-AKT and PARP protein expression levels after apatinib and docetaxel treatment. (B) LC3A mRNA expression after apatinib and docetaxel treatment. (C) Beclin-1 mRNA expression after apatinib and docetaxel treatment. (D) Representative flow cytometry images of cell apoptosis and (E) bar graph presenting the cell apoptosis rate following treatment with apatinib and docetaxel. ${ }^{*} \mathrm{P}<0.05,{ }^{* *} \mathrm{P}<0.01$ and ${ }^{* * * *} \mathrm{P}<0.001$. LC $3 \mathrm{~A}$, light chain $3 \alpha$; p-AKT, phosphorylated AKT; PARP, poly (ADP) ribose polymerase.

A

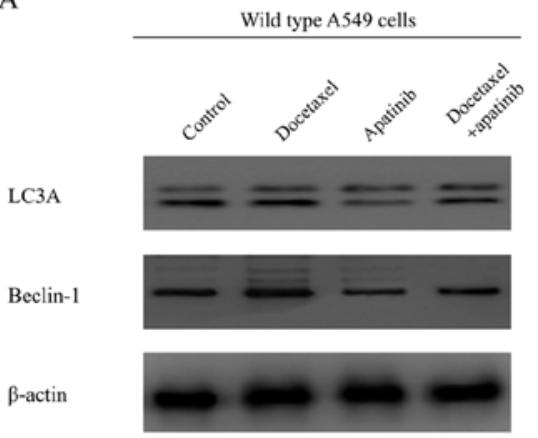

B
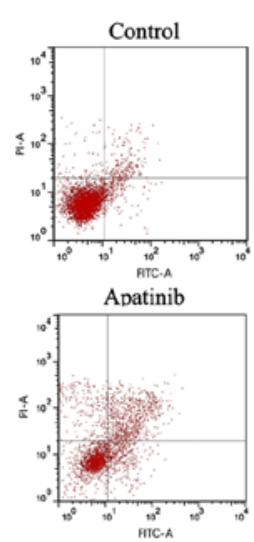
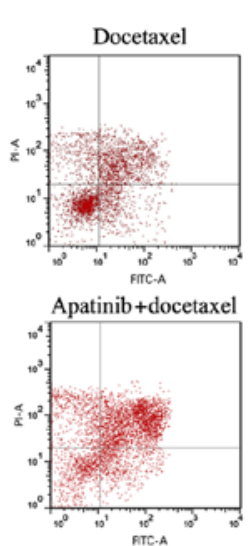

C

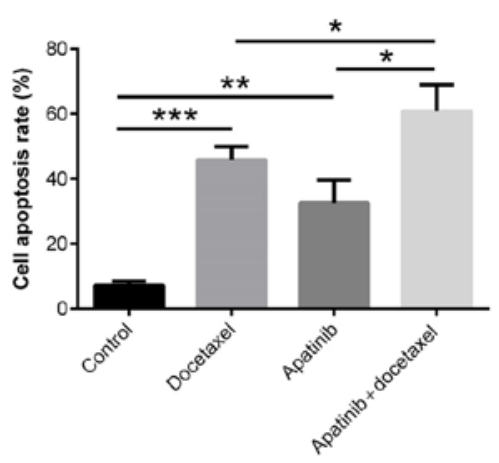

Figure 5. LC3A and Beclin-1 expression levels, and cell apoptosis rate of wild-type A549 cells following apatinib and docetaxel treatment. (A) LC3A and Beclin-1 expression levels after apatinib and docetaxel treatment. (B) Representative flow cytometry images of cell apoptosis and (C) bar graph presenting the cell apoptosis rate following treatment with apatinib and docetaxel. ${ }^{*} \mathrm{P}<0.05,{ }^{* *} \mathrm{P}<0.01$ and ${ }^{* * * *} \mathrm{P}<0.001$. LC $3 \mathrm{~A}$, light chain $3 \alpha$.

Anti-angiogenesis drugs plus chemotherapy have been introduced to treat several types of cancer at the advanced stage, including advanced/metastatic urothelial carcinoma, advanced gastric cancer and advanced NSCLC, as second-line treatments or beyond (23-25). For instance, ramucirumab plus docetaxel prolongs progression-free survival and overall survival compared with docetaxel alone in patients with stage IV NSCLC, when administered during or after a first-line platinum-based chemotherapy regimen (25). As the first independently developed small-molecule anti-angiogenesis 
agent in China, apatinib has attracted increased attention in the oncology field (4). Previous studies examining apatinib plus chemotherapy in treating advanced cancer have focused on cancer types including gastric, colorectal and ovarian cancer, while studies reporting on its use in treating NSCLC are currently limited $(8,9)$. For instance, in a single-center, open-label, dose-escalating phase I trial it was observed that apatinib plus docetaxel was well tolerated and exhibited promising efficacy in patients with advanced lung adenocarcinoma; however, the study only included 12 patients and lacked a control group who only receive docetaxel (8). Another multi-center, prospective study revealed that apatinib plus docetaxel achieved an ORR of $33 \%$ and a DCR of $67 \%$ in the treatment of patients with advanced non-squamous NSCLC, but the study only included 14 patients and also lacked a group treated with docetaxel as a control (9).

In the present study, in order to assess the efficacy and safety of apatinib plus docetaxel in treating advanced NSCLC, 39 patients with primary NSCLC at stage IV or recurrent metastatic NSCLC refractory/intolerant to standard therapy were enrolled and treated with apatinib plus docetaxel or with docetaxel alone. The results indicated that apatinib plus docetaxel achieved a higher ORR (37 vs. 10\%) and DCR (84 vs. $45 \%$ ) compared with docetaxel alone in these patients. Furthermore, in the tumor tissue of patients treated with docetaxel, the expression levels of Beclin-1 and LC3A were increased post-treatment compared with to those prior to treatment, while these remained unchanged in the apatinib plus docetaxel-treated group. Thus, it was suggested that apatinib may enhance the efficacy of docetaxel via attenuating autophagy in patients with advanced NSCLC. A possible explanation for the present findings is that autophagy significantly contributes to the resistance of NSCLC to docetaxel, while apatinib inhibits autophagy via regulating pathways, including AKT/mTOR and VEGFR2/STAT3/Bcl-2, in cancer (15-17), and therefore, its application enhances the efficacy of docetaxel in patients with advanced NSCLC. This hypothesis was further evaluated via the subsequent in vitro experiments of the present study. Moreover, as previously indicated, apatinib increases the uptake of docetaxel into NSCLC cells and drug-resistant NSCLC cells (19), which may explain how apatinib improved the treatment outcome of docetaxel in patients with advanced NSCLC in the current study.

Docetaxel resistance is a critical issue in treating advanced NSCLC, and its potential underlying mechanisms have been elucidated, including the implication of intracellular multidrug resistance-associated protein P-glycoprotein, lung resistance protein, glutathione transferase and t-structural changes of intracellular microtubules topoisomerase (26). In addition, it has been reported that high-mobility group box 1-mediated autophagy contributes to docetaxel resistance in lung adenocarcinoma (27), while another study revealed that Klotho-mediated autophagy was closely involved in chemotherapy resistance (including docetaxel resistance) in lung cancer (28). In the present study, it was also demonstrated that autophagy attenuated the cytotoxic effects of docetaxel on A549/DTX cells, which was in line with these previous findings.

With regards to the autophagy regulation of apatinib, this drug was previously reported to regulate autophagy via controlling the AKT/mTOR pathway in anaplastic thyroid cancer (15). Moreover, apatinib can modulate autophagy markers and the AKT/mTOR pathway in colon cancer (16), as well as regulate autophagy and apoptosis of osteosarcoma cells via the VEGFR2/STAT3/Bcl-2 signaling pathway (17). However, to the best of our knowledge, its regulation of autophagy in NSCLC has not been previously reported. The present results suggested that in the Docetaxel group, Beclin-1 and LC3A expression levels were increased in NSCLC tumor tissues post-treatment compared with that prior to treatment, while these remained unchanged in the Apatinib plus docetaxel group. Therefore, it was speculated that apatinib may also regulate autophagy in docetaxel-treated patients with advanced NSCLC. Furthermore, the results of the in vitro experiments indicated that autophagy attenuated the cytotoxic effect of docetaxel on A549/DTX cells. Thus, the effect of apatinib to regulate autophagy in the presence of docetaxel in A549/DTX cells was further investigated, which demonstrated that apatinib sensitized A549/DTX cells to the cytotoxic effects of docetaxel via repressing autophagy. The possible mechanism may be that apatinib inhibits autophagy via regulating the AKT/mTOR and VEGFR2/STAT3/Bcl-2 pathways (15-17), leading to an increase in the anti-cancer efficacy of docetaxel. However, the detailed molecular mechanisms underlying apatinib-mediated inhibition of autophagy were not investigated in the present study, which was a limit of the present study and requires further investigation.

In conclusion, apatinib synergizes the effect of docetaxel in treating patients with advanced NSCLC or recurrent/refractory NSCLC, as well as chemoresistant NSCLC cells via regulating autophagy. The present findings may provide novel evidence for the combined application of apatinib and docetaxel in treating advanced NSCLC and recurrent/refractory NSCLC.

\section{Acknowledgements}

Not applicable.

\section{Funding}

This study was supported by National Key Research and Development Program of China (grant no. 2018YFC1313602), Major International (Regional) Joint Research Project (grant no. 81820108001), National Natural Science Foundation of China (81670029), Jiangsu Key Principal Investigator of Medicine (grant no. ZDRCA2016018), Project 333 for Cultivation of Young and Middle-aged Leading Talents (grant no. BRA2019078) and Jiangsu Key Program of Social Development (grant no. BE2015651).

\section{Availability of data and materials}

The datasets used and/or analyzed during the current study are available from the corresponding author on reasonable request.

\section{Authors' contributions}

LZ conceived and designed the experiment, analyzed the data and revised the manuscript. RH, TL and KH collected 
the data and analyzed the data. ZC and NW performed data analysis and provided interpretation. XW provided technical support, and analyzed and interpreted the results. LG critically revised the article and interpreted the data. All authors read and approved the final manuscript.

\section{Ethics approval and consent to participate}

This study was approved by the Ethics Committee of The First Affiliated Hospital of Nanjing Medical University (Nanjing, China) and all patients signed the informed consent before the enrollment.

\section{Patient consent for publication}

Not applicable.

\section{Competing interests}

The authors declare that they have no competing interests.

\section{References}

1. Chen W, Zheng R, Baade PD, Zhang S, Zeng H, Bray F, Jemal A, Yu XQ and He J: Cancer statistics in China, 2015. CA Cancer J Clin 66: 115-132, 2016.

2. Hirsch FR, Scagliotti GV, Mulshine JL, Kwon R, Curran WJ Jr, Wu YL and Paz-Ares L: Lung cancer: Current therapies and new targeted treatments. Lancet 389: 299-311, 2017.

3. Tanino R, Tsubata Y, Harashima N, Harada M and Isobe T: Novel drug-resistance mechanisms of pemetrexed-treated non-small cell lung cancer. Oncotarget 9: 16807-16821, 2018.

4. Tian S, Quan H, Xie C, Guo H, Lü F, Xu Y, Li J and Lou L: YN968D1 is a novel and selective inhibitor of vascular endothelial growth factor receptor-2 tyrosine kinase with potent activity in vitro and in vivo. Cancer Sci 102: 1374-1380, 2011.

5. Li J, Zhao X, Chen L, Guo H, Lv F, Jia K, Yv K, Wang F, Li C, Qian J, et al: Safety and pharmacokinetics of novel selective vascular endothelial growth factor receptor-2 inhibitor YN968D1 in patients with advanced malignancies. BMC Cancer 10: 529, 2010

6. Maroufi NF, Rashidi MR, Vahedian V, Akbarzadeh M, Fattahi A and Nouri M: Therapeutic potentials of apatinib in cancer treatment: Possible mechanisms and clinical relevance. Life Sci 241: 117106, 2020.

7. Wu F, Zhang S, Gao G, Zhao J, Ren S and Zhou C: Successful treatment using apatinib with or without docetaxel in heavily pretreated advanced non-squamous non-small cell lung cancer: A case report and literature review. Cancer Biol Ther 19: 141-144, 2018.

8. Duan JC, Wang ZJ, Lin L, Li JL, Wang Y, Bai H, Hu XS, Liu YT, Hao XZ, Wang HY et al: Apatinib, a novel VEGFR inhibitor plus docetaxel in advanced lung adenocarcinoma patients with wild-type EGFR: A phase I trial. Invest New Drugs 37: 731-737, 2019.

9. Jiang Q, Zhang NL, Ma DY, Tan BX, Hu X and Fang XD: Efficacy and safety of apatinib plus docetaxel as the second or above line treatment in advanced nonsquamous NSCLC: A multi center prospective study. Medicine (Baltimore) 98: e16065, 2019.

10. Mi YJ, Liang YJ, Huang HB, Zhao HY, Wu CP, Wang F, Tao LY, Zhang CZ, Dai CL, Tiwari AK, et al: Apatinib (YN968D1) reverses multidrug resistance by inhibiting the efflux function of multiple ATP-binding cassette transporters. Cancer Res 70: 7981-7991, 2010.

11. Li J, Jia Y, Gao Y, Chang Z, Han H, Yan J and Qin Y: Clinical efficacy and survival analysis of apatinib combined with docetaxel in advanced esophageal cancer. Onco Targets Ther 12: 2577-2583, 2019.

12. Li YJ, Lei YH, Yao N, Wang CR, Hu N, Ye WC, Zhang DM and Chen ZS: Autophagy and multidrug resistance in cancer. Chin J Cancer 36: 52, 2017.
13. Azam F, Latif MF, Farooq A, Tirmazy SH, AlShahrani S, Bashir S and Bukhari N: Performance status assessment by using ECOG (Eastern Cooperative Oncology Group) score for cancer patients by oncology healthcare professionals. Case Rep Oncol 12: 728-736, 2019.

14. Lencioni R and Llovet JM: Modified RECIST (mRECIST) assessment for hepatocellular carcinoma. Semin Liver Dis 30: 52-60, 2010.

15. Feng H, Cheng X, Kuang J, Chen L, Yuen S, Shi M, Liang J, Shen B, Jin Z, Yan J and Qiu W: Apatinib-induced protective autophagy and apoptosis through the AKT-mTOR pathway in anaplastic thyroid cancer. Cell Death Dis 9: 1030, 2018.

16. Lu W, Ke H, Qianshan D, Zhen W, Guoan X and Honggang Y: Apatinib has anti-tumor effects and induces autophagy in colon cancer cells. Iran J Basic Med Sci 20: 990-995, 2017.

17. Liu K, Ren T, Huang Y, Sun K, Bao X, Wang S, Zheng B and Guo W: Apatinib promotes autophagy and apoptosis through VEGFR2/STAT3/BCL-2 signaling in osteosarcoma. Cell Death Dis 8: e3015, 2017.

18. Fu H, Jin C, Zhu Q, Liu T, Ke B, Li A and Zhang T: Dysregulated expressions of PTEN, NF-kB, WWP2, p53 and c-Myc in different subtypes of B cell lymphoma and reactive follicular hyperplasia. Am J Transl Res 11: 1092-1101, 2019.

19. Feng SQ, Wang GJ, Zhang JW, Xie Y, Sun RB, Fei F, Huang JQ, Wang Y, Aa JY and Zhou F: Combined treatment with apatinib and docetaxel in A549 xenograft mice and its cellular pharmacokinetic basis. Acta Pharmacol Sin 39: 1670-1680, 2018.

20. Liang L, Hui K, Hu C, Wen Y, Yang S, Zhu P, Wang L, Xia Y, Qiao Y, Sun W, et al: Autophagy inhibition potentiates the anti-angiogenic property of multikinase inhibitor anlotinib through JAK2/STAT3/VEGFA signaling in non-small cell lung cancer cells. J Exp Clin Cancer Res 38: 71, 2019.

21. Bulatov E, Sayarova R, Mingaleeva R, Miftakhova R, Gomzikova M, Ignatyev Y, Petukhov A, Davidovich P, Rizvanov A and Barlev NA: Isatin-schiff base-copper (II) complex induces cell death in p53-positive tumors. Cell Death Discov 4: 103, 2018.

22. Livak KJ and Schmittgen TD: Analysis of relative gene expression data using real-time quantitative PCR and the 2(-Delta Delta C(T)) method. Methods 25: 402-408, 2001.

23. Petrylak DP, de Wit R, Chi KN, Drakaki A, Sternberg CN, Nishiyama H, Castellano D, Hussain SA, Fléchon A, Bamias A, et al: Ramucirumab plus docetaxel versus placebo plus docetaxel in patients with locally advanced or metastatic urothelial carcinoma after platinum-based therapy (RANGE): Overall survival and updated results of a randomised, double-blind, phase 3 trial. Lancet Oncol 21: 105-120, 2020.

24. Takahari D: Second-line chemotherapy for patients with advanced gastric cancer. Gastric Cancer 20: 395-406, 2017.

25. Garon EB, Ciuleanu TE, Arrieta O, Prabhash K, Syrigos KN, Goksel T, Park K, Gorbunova V, Kowalyszyn RD, Pikiel J, et al: Ramucirumab plus docetaxel versus placebo plus docetaxel for second-line treatment of stage IV non-small-cell lung cancer after disease progression on platinum-based therapy (REVEL): A multicentre, double-blind, randomised phase 3 trial. Lancet 384: 665-673, 2014.

26. Chen ZJ, Le HB, Zhang YK, Qian LY, Sekhar KR and Li WD: Lung resistance protein and multidrug resistance protein in non-small cell lung cancer and their clinical significance. J Int Med Res 39: 1693-1700, 2011.

27. Pan B, Chen D, Huang J, Wang R, Feng B, Song H and Chen L: HMGB1-mediated autophagy promotes docetaxel resistance in human lung adenocarcinoma. Mol Cancer 13: 165, 2014.

28. Chen T, Ren H, Thakur A, Yang T, Li Y, Zhang S, Wang T and Chen MW: Decreased level of klotho contributes to drug resistance in lung cancer cells: Involving in klotho-mediated cell autophagy. DNA Cell Biol 35: 751-757, 2016.

This work is licensed under a Creative Commons Attribution-NonCommercial-NoDerivatives 4.0 International (CC BY-NC-ND 4.0) License. 